\title{
CONSTRUCTION OF MAXIMAL UNRAMIFIED $p$-EXTENSIONS WITH PRESCRIBED GALOIS GROUPS
}

\author{
MANABU OZAKI
}

\section{INTRODUCTION}

For any number field $F$ (not necessary of finite degree) and prime number $p$, let $F^{\mathrm{ur}, p}$ denote the maximal unramified $p$-extension over $F$, and put $\widetilde{G}_{F}(p)=\operatorname{Gal}\left(F^{\mathrm{ur}, p} / F\right)$. Though the structure of $\tilde{G}_{F}(p)$ has been one of the most fascinating theme of number theory, our knowledge on it is not enough even at present: It had been a cerebrated open problem for a long time whether $\widetilde{G}_{F}(p)$ can be infinite for a number field $F$ of finite degree, and Golod and Shafarevich solved it by giving $F$ with infinite $\widetilde{G}_{F}(p)$. Hence we barely know that $\widetilde{G}_{F}(p)$ can be infinite. However we do not know exactly what kind of pro- $p$ groups occur as $\widetilde{G}_{F}(p)$; for example, there are no examples of infinite $\widetilde{G}_{F}(p)$ for number fields $F$ of finite degree whose structure is completely determined. The known general property of the group $\widetilde{G}_{F}(p)$ for number fields $F$ of finite degree is only that $\widetilde{G}_{F}(p)$ is a finitely presented pro- $p$-group any whose open subgroup has finite abelianization, which comes from rather fundamental facts of algebraic number theory, namely, class field theory and the finiteness of the ideal class group. Indeed, a consequence of the Fontaine-Mazur conjecture predicts that $\widetilde{G}_{F}(p)$ has a certain distinguished property (see [1]), however, this conjecture seems far reach object at present.

On the other hand, we have known that many kind of pro-p-groups, especially, finite $p$-groups in fact occur as $\widetilde{G}_{F}(p)$. For example, Scholz and Taussky [4] have already determined the structure of $\widetilde{G}_{F}(p)$ for $p=3$ and $F=\mathbb{Q}(\sqrt{-4027})$ in 1930's : this group is a non-abelian finite group of oder $3^{5}$. Also, Yahagi [7] showed that for any given finite abelian $p$-group $A$ there exists an number field $F$ of finite degree such that $\widetilde{G}_{F}(p)^{\mathrm{ab}} \simeq A$.

In the present paper, we shall show that every finite $p$-group occurs as $\widetilde{G}_{F}(p)$ for a certain number field $F$ of finite degree under a technical assumption on the prime number $p$ :

Theorem 1. Let $p$ be a prime number satisfying the following condition, say $\mathrm{C}(p)$ :

2000 Mathematics Subject Classification. 11R32,

This research is partially supported by the Grant-in-Aid for Young Scientists (B), Ministry of Education, Science, Sports and Culture, Japan. 
the maximal unramified $p$-extension over the $p$-th cyclotomic field $\mathbb{Q}\left(\mu_{p}\right)$ is finite.

Then for any given finite $p$-group $G$, there exists a number field $F$ of finite degree such that $\widetilde{G}_{F}(p) \simeq G$.

We note here that if $p$ is a regular prime or the $p$-primary part of the class group of $\mathbb{Q}\left(\mu_{p}\right)$ is cyclic, then the prime $p$ satisfies condition $\mathrm{C}(p)$. Hence all the prime numbers less than 157 satisfy condition $\mathrm{C}(p)$. Also, we have no example of prime numbers $p$ which do not satisfy condition $\mathrm{C}(p)$ at present.

In the case where we allow a number field $F$ to be of infinite degree, we obtain the following:

Theorem 2. Let $p$ be a prime number satisfying the above condition $\mathrm{C}(p)$. Then for any given pro- $p$-group $G$ with countably many generators, there exists a number field $F$ (not necessary of finite degree) such that $\widetilde{G}_{F}(p) \simeq G$.

Since the Galois group of a pro- $p$-extension of number fields must have countably many generators, Theorem 2 says that every possible pro- $p$-group could occur as the Galois group of the maximal unramified $p$-extension over a certain number field under condition $\mathrm{C}(p)$.

\section{Strategy of Construction} 2.

It is enough to show the following two propositions to prove Theorems 1 and

Proposition 1. Let $p$ be a prime number, and let $k$ be a number field of finite degree satisfying the following conditions:

(i) the field $k$ contains the group $\mu_{p}$ of all the $p$-th roots of unity and is totally imaginary if $p=2$

(ii) $G:=\widetilde{G}_{k}(p)$ is finite and $[k: \mathbb{Q}] \geq 2(2 d(G)+r(G))$, where $d(G)=$ $\operatorname{dim}_{\mathbb{F}_{p}} H^{1}(G, \mathbb{Z} / p)$ and $r(G)=\operatorname{dim}_{\mathbb{F}_{p}} H^{2}(G, \mathbb{Z} / p)$ denote the generator rank and the relation rank of $G$, respectively,

(iii) every prime of $k$ lying over $p$ splits completely in $k^{\mathrm{ur}, p}$.

Then there exists a cyclic extension $k^{\prime} / k$ of degree $p$ such that $k^{\prime} \cap k^{\mathrm{ur}, p}=k$, $\left(k^{\prime}\right)^{\mathrm{ur}, p}=k^{\prime} k^{\mathrm{ur}, p}$, and $\widetilde{G}_{k^{\prime}}(p) \simeq G$.

Proposition 2. Let $p$ be a prime number, and let $k$ be a number field of finite degree satisfying conditions (i)-(iii) of Proposition 1, Then for any given exact sequence

$$
1 \longrightarrow \mathbb{Z} / p \longrightarrow G^{\prime} \longrightarrow \widetilde{G}_{k}(p) \longrightarrow 1
$$

of finite $p$-groups, there exists a finite extension $K / k$ such that $\widetilde{G}_{K}(p) \simeq$ $G^{\prime}, K \cap k^{\mathrm{ur}, p}=k$ and every prime of $K$ lying over $p$ splits completely in $K^{\text {ur, } p}$

One can derive Theorem 1 from Propositions 1 and 2 as follows: We first remark that condition $\mathrm{C}(p)$ is equivalent to that there exists a number field 
$k$ of finite degree such that $\mu_{p} \subseteq k$ and $\widetilde{G}_{k}(p)=1\left(k=\mathbb{Q}\left(\mu_{p}\right)^{\text {ur, } p}\right.$ has this property under $\mathrm{C}(p))$.

For any finite $p$-group $G$, there exists a sequence of finite $p$-groups with surjections

$$
1=G_{0} \longleftarrow G_{1} \longleftarrow \cdots \longleftarrow G_{n} \longleftarrow G_{n+1} \longleftarrow \cdots \longleftarrow G_{r-1} \longleftarrow G_{r}=G
$$

such that $\operatorname{ker}\left(G_{n+1} \longrightarrow G_{n}\right) \simeq \mathbb{Z} / p$ for each $n$. It follows from the above remark that there exists a number field $k_{0}$ of finite degree such that $k_{0}$ satisfies conditions (i)-(iii) and $\widetilde{G}_{k_{0}}(p) \simeq G_{0}=1$ because $d\left(G_{0}\right)=r\left(G_{0}\right)=0$. Assume that there exists a number field $k_{n}(n \leq r-1)$ which satisfies conditions (i)-(iii) and $\widetilde{G}_{k_{n}}(p) \simeq G_{n}$. Then by using Proposition11repeatedly, we see that $k_{n}$ has a finite extension field $k_{n}^{\prime}$ such that $\widetilde{G}_{k_{n}^{\prime}}(p) \simeq G_{n}, k_{n}^{\prime} \cap k_{n}^{\text {ur, } p}=k_{n}$, and $\left[k_{n}^{\prime}: \mathbb{Q}\right] \geq$ $2\left(2 d\left(G_{n+1}\right)+r\left(G_{n+1}\right)\right)$. Since this field $k_{n}^{\prime}$ also satisfies conditions (i)-(iii), it follows from Proposition 2 that there exists a finite extension $k_{n+1} / k_{n}^{\prime}$ such that $\widetilde{G}_{k_{n+1}}(p) \simeq G_{n+1}, k_{n+1} \cap k_{n}^{\mathrm{ur}, p}=k_{n}$, and $k_{n+1}$ satisfies conditions (i)-(iii). Therefore we obtain field $F:=k_{r}$ with $\widetilde{G}_{F}(p) \simeq G_{r}=G$ by induction. This proves Theorem 1 .

Theorem 2 also follows similarly to the above: We may assume that $G$ is infinite. For any infinite pro- $p$ group $G$ with countably many generators, there exists a projective system of finite $p$-groups $G_{n}(n \in \mathbb{N})$ and surjective morphisms $\phi_{m, n}: G_{m} \rightarrow G_{n}(m \geq n)$ such that $G$ is isomorphic to the projective $\operatorname{limit} \lim G_{n}$ of this projective system and $\operatorname{ker} \phi_{n+1, n} \simeq \mathbb{Z} / p$. Hence we can construct a tower of number fields $k_{n}$ of finite degree

$$
k_{0} \subseteq k_{1} \subseteq \cdots \subseteq k_{n} \subseteq k_{n+1} \subseteq \cdots \subseteq F:=\bigcup_{n \in \mathbb{N}} k_{n}
$$

such that $\widetilde{G}_{k_{n}}(p) \simeq G_{n}$ and $k_{n}^{\mathrm{ur}, p} \cap F=k_{n}$ as in the above proof of Theorem 1 , from which we deduce the commutative diagram

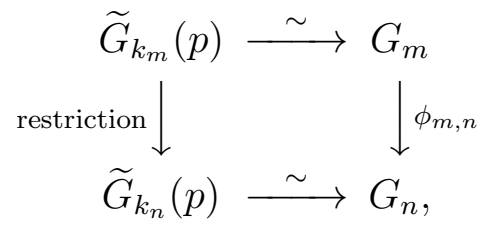

with suitable isomorphisms $\widetilde{G}_{k_{m}}(p) \simeq G_{m}$. Then $F$ is a desired field because $F^{\mathrm{ur}, p}=\bigcup_{n \in \mathbb{N}} k_{n}^{\mathrm{ur}, p}$ and $\widetilde{G}_{F}(p) \simeq \lim _{\longleftarrow} \widetilde{G}_{k_{n}}(p) \simeq G$.

\section{Proof of Proposition 1}

In this section, we give a proof of Proposition 1. Let $p$ be a prime number , and let $k$ be a number field with properties (i)-(iii) of the proposition for this prime number $p$.

We introduce some notations which will be used in what follows. For any number field $F$ of finite degree and prime $\mathfrak{p}$ of $F$ lying above $p$, we denote by $U_{\mathfrak{p}}(F)$ the pro- $p$-part of the local unit group of the completed field $F_{\mathfrak{p}}$ of $F$ at $\mathfrak{p}$, and define the pro- $p$-part of the semi-local unit group at $p$ by 
$U(F)=\bigoplus_{\mathfrak{p} \mid p} U_{\mathfrak{p}}(F)$. We embed the unit group of the localization of the maximal order $\mathcal{O}_{F}$ of $F$ at $p$ diagonally into $U(F)$ as usual. Define $U_{\mathfrak{p}}^{\prime}(F)$ to be the submodule of $U_{\mathfrak{p}}(F)$ consisting of all the elements $u \in U_{\mathfrak{p}}(F)$ such that $F_{\mathfrak{p}}(\sqrt[p]{u}) / F_{\mathfrak{p}}$ is an unramified extension (including the case $F_{\mathfrak{p}}(\sqrt[p]{u})=F_{\mathfrak{p}}$ ) for a prime $\mathfrak{p}$ lying over $p$, and put $U^{\prime}(F)=\bigoplus_{\mathfrak{p} \mid p} U_{\mathfrak{p}}^{\prime}(F)$. Then we have the inclusions $U(F)^{p} \subseteq U^{\prime}(F) \subseteq U(F)$ and put $R(F)=U(F) / U(F)^{p}$ and $R^{\prime}(F)=U(F) / U^{\prime}(F)$.

It follows from the assumption $\mu_{p} \subseteq k$ that $R(k) \simeq(\mathbb{Z} / p)^{\oplus[k: \mathbb{Q}]+s}$ and $R^{\prime}(k) \simeq$ $(\mathbb{Z} / p)^{\oplus[k: \mathbb{Q}]}$ as $\mathbb{Z}_{p}$-modules if $s$ denotes the number of the primes of $k$ lying over $p$. Because every prime of $k$ lying over $p$ splits completely in $k^{\mathrm{ur}, p} / k$, we have the natural isomorphisms of $G$-modules $\left(G=\operatorname{Gal}\left(k^{\mathrm{ur}, p} / k\right)\right) U\left(k^{\mathrm{ur}, p}\right) \simeq$ $\mathbb{Z}_{p}[G] \otimes_{\mathbb{Z}_{p}} U(k)$ and $U^{\prime}\left(k^{\mathrm{ur}, p}\right) \simeq \mathbb{Z}_{p}[G] \otimes_{\mathbb{Z}_{p}} U^{\prime}(k)$, from which we derive

$$
R\left(k^{\mathrm{ur}, p}\right) \simeq \mathbb{F}_{p}[G]^{\oplus[k: \mathbb{Q}]+s}, \quad R^{\prime}\left(k^{\mathrm{ur}, p}\right) \simeq \mathbb{F}_{p}[G]^{\oplus[k: \mathbb{Q}]}
$$

as $G$-modules.

In what follow, we find out an integer $\lambda$ of $k$ such that $\lambda \mathcal{O}_{k}$ is a prime ideal of $k, \mathcal{O}_{k}$ being the maximal order of $k$, and that $k^{\mathrm{ur}, p}(\sqrt[p]{\lambda})$ has no non-trivial unramified $p$-extensions. Then the cyclic extension field $k^{\prime}=k(\sqrt[p]{\lambda})$ of $k$ has desired properties with $\left(k^{\prime}\right)^{\mathrm{ur}, p}=k^{\mathrm{ur}, p}(\sqrt[p]{\lambda})$.

Lemma 1. Let $p$ be any prime number, $F$ a number field of finite degree with $F^{\mathrm{ur}, p}=F$ and $S$ a finite set of primes of $F$. We denote by $F_{S} / F$ the maximal elementary abelian $p$-extension unramified outside $S$. For any prime $v$ of $F$, we write $D_{v}$ for the decomposition subgroup of $\operatorname{Gal}\left(F_{S} / F\right)$ at the prime $v$. We assume that the map

$$
\phi: \bigoplus_{v: \text { prime of } F} H_{2}\left(D_{v}, \mathbb{Z}\right) \longrightarrow H_{2}\left(\operatorname{Gal}\left(F_{S} / F\right), \mathbb{Z}\right)
$$

induced by the natural inclusions $D_{v} \subseteq \operatorname{Gal}\left(F_{S} / F\right)$ is surjective. Then we have $F_{S}^{\mathrm{ur}, p}=F_{S}$.

Corollary 1. Under the assumption of Lemma 1, if $F_{S} / F$ is a cyclic extension, then $F_{S}^{\mathrm{ur}, p}=F_{S}$.

Proof of Lemma 1. We first note that $F_{S} / F$ is a finite extension. Let $M$ be the genus $p$-class field of $F_{S} / F$, namely, the maximal unramified $p$-extension $M$ of $F_{S}$ such that there exists an abelian extension $F^{\prime} / F$ with $M=F_{S} F^{\prime}$. Also, we denote by $L$ the central $p$-class field of $F_{S} / F$, namely, $L / F$ is the maximal normal sub-extension of $F_{S}^{\mathrm{ur}, p} / F$ containing $F_{S}$ such that $\operatorname{Gal}\left(L / F_{S}\right)$ is contained in the center of $\operatorname{Gal}(L / F)$. In another word, $L$ is the fixed field of $F_{S}^{\mathrm{ur}, p}$ by the (topological) commutator subgroup $\left(\operatorname{Gal}\left(F_{S}^{\mathrm{ur}, p} / F_{S}\right), \operatorname{Gal}\left(F_{S}^{\mathrm{ur}, p} / F\right)\right)$ of $\operatorname{Gal}\left(F_{S}^{\mathrm{ur}, p} / F_{S}\right)$. Then $M$ is contained in $L$ and we have a surjection

$$
\operatorname{coker} \phi \longrightarrow \operatorname{Gal}(L / M)
$$

(see Thm. 3.11 in [2] and section 11.4 in [5]). Hence we have $M=L$ by the assumption coker $\phi=0$. Suppose that $F_{S} F^{\prime} / F_{S}$ is a non-trivial unramified 
p-extension for an abelian extension $F^{\prime} / F$. Then it follows from the definition of $F_{S}$ that $F^{\prime} / F$ is unramified outside $S$, and that $F^{\prime}$ must contain a cyclic extension $F_{0} / F$ of degree $p^{2}$ over $F$, which is totally ramified at some ramified prime by $F^{\mathrm{ur}, p}=F$. Hence $F_{S} F^{\prime} / F_{S}$ is not unramified, which is a contradiction. Thus we conclude that $L=M=F_{S}$.

Since $\operatorname{Gal}\left(F_{S}^{\mathrm{ur}, p} / F\right)$ is a pro- $p$-group, it follows from the isomorphism

$$
1=\operatorname{Gal}\left(L / F_{S}\right) \simeq \operatorname{Gal}\left(F_{S}^{\mathrm{ur}, p} / F_{S}\right) /\left(\operatorname{Gal}\left(F_{S}^{\mathrm{ur}, p} / F_{S}\right), \operatorname{Gal}\left(F_{S}^{\mathrm{ur}, p} / F\right)\right),
$$

that $F_{S}^{\mathrm{ur}, p}=F_{S}$. Therefore we obtain the lemma. The corollary follows from the lemma since $H_{2}\left(\operatorname{Gal}\left(F_{S} / F\right), \mathbb{Z}\right)=0$ if $\operatorname{Gal}\left(F_{S} / F\right)$ is a cyclic group.

Now we shall show that there exists an integer $\Lambda$ of $k^{\mathrm{ur}, p}$ such that

(a) $\Lambda \mathcal{O}_{k^{\mathrm{ur}, p}}$ is a prime ideal of degree 1 , not lying over $p$,

(b) if $S$ denotes the set of primes of $k^{\mathrm{ur}, p}$ dividing $\lambda:=N_{k^{\mathrm{ur}, p} / k} \Lambda$, then $k^{\mathrm{ur}, p}(\sqrt[p]{\lambda})$ is the maximal elementary abelian $p$-extension over $k^{\mathrm{ur}, p}$ unramified outside $S$.

Then $k^{\prime}=k(\sqrt[p]{\lambda})$ is a desired extension of $k$, because $k^{\mathrm{ur}, p}(\sqrt[p]{\lambda})$ has no nontrivial unramified $p$-extension by Corollary 1 and the fact $\left(k^{\mathrm{ur}, p}\right)^{\mathrm{ur}, p}=k^{\mathrm{ur}, p}$ (Note that $\lambda \mathcal{O}_{k}$ is a prime ideal of $k$, hence $k(\sqrt[p]{\lambda}) / k$ is of degree $p$ and $k^{\mathrm{ur}, p} \cap$ $k(\sqrt[p]{\lambda})=k)$. Assume that $\Lambda \in k^{\mathrm{ur}, p}$ has property (a) above. We see that if $k^{\mathrm{ur}, p}(\sqrt[p]{\alpha}) / k^{\mathrm{ur}, p}$ is unramified outside $S$ for $\alpha \in k^{\mathrm{ur}, p}$, then

$$
\alpha \bmod \left(k^{\mathrm{ur}, p \times}\right)^{p}=\left(\varepsilon \bmod \left(k^{\mathrm{ur}, p \times}\right)^{p}\right)+\xi\left(\Lambda \bmod \left(k^{\mathrm{ur}, p \times}\right)^{p}\right)
$$

for some $\varepsilon \in \mathcal{O}_{k^{\mathrm{ur}, p}}^{\times}$and $\xi \in \mathbb{F}_{p}[G]$ such that

$$
\left(\varepsilon \bmod U^{\prime}\left(k^{\mathrm{ur}, p}\right)\right)+\xi\left(\Lambda \bmod U^{\prime}\left(k^{\mathrm{ur}, p}\right)\right)=0
$$

(We shall use additive notation for the group operation on the multiplicative groups $F^{\times} /\left(F^{\times}\right)^{p}, R(F)$ and $R^{\prime}(F)$ for a number field $F$ ). Hence if (3) holds only for $\varepsilon \in\left(\mathcal{O}_{k^{\mathrm{ur}, p}}^{\times}\right)^{p}$ and $\xi \in \mathbb{F}_{p} N_{G}, N_{G}=\sum_{\sigma \in G} \sigma$, then condition (b) also holds for this $\Lambda$ (Here we need not take care of ramification at the archimedean primes since $k$ is totally imaginary by the assumption).

Let $E=E\left(k^{\mathrm{ur}, p}\right)$ be the image of the natural map $\mathcal{O}_{k^{\mathrm{ur}, p}, p}^{\times} \longrightarrow R^{\prime}\left(k^{\mathrm{ur}, p}\right)$. Since $k^{\mathrm{ur}, p}(\sqrt[p]{\varepsilon}) / k^{\mathrm{ur}, p}$ is ramified at some prime lying over $p$ for any $\varepsilon \in \mathcal{O}_{k^{\mathrm{ur}, p}}^{\times}$ $\left(\mathcal{O}_{k^{\mathrm{ur}, p}}^{\times}\right)^{p}$, the above map is injective and $E \simeq \mathcal{O}_{k^{\mathrm{ur}, p}}^{\times} / p$. Hence we see that the natural map $\mathcal{O}_{k^{\mathrm{ur}, p}, p}^{\times} \longrightarrow R\left(k^{\mathrm{ur}, p}\right)$ is also injective. We also denote by the same letter $E$ the image of this map.

Lemma 2. Let $N$ be the kernel of the natural projection map $\pi: R\left(k^{\mathrm{ur}, p}\right) \longrightarrow$ $R^{\prime}\left(k^{\mathrm{ur}, p}\right)$. Then there exist free $\mathbb{F}_{p}[G]$-submodules $P$ and $Q$ of $R\left(k^{\mathrm{ur}, p}\right)$ such that

$$
R\left(k^{\mathrm{ur}, p}\right)=P \oplus Q \oplus N, R^{\prime}\left(k^{\mathrm{ur}, p}\right) \stackrel{\left(\left.\pi\right|_{P \oplus Q}\right)^{-1}}{\simeq} P \oplus Q
$$

and that $E \subseteq P$ and $\operatorname{rank}_{\mathbb{F}_{p}[G]} Q \geq \frac{1}{2}[k: \mathbb{Q}]-d(G)-r(G)$.

Proof. For any $\mathbb{F}_{p}[G]$-module $A$, we put $A^{*}=\operatorname{Hom}_{\mathbb{F}_{p}}\left(A, \mathbb{F}_{p}\right)$ and define left $G$-action on it by $\sigma f:=f \circ \sigma^{-1}$ for $f \in A^{*}$ and $\sigma \in G$. Then one can show that $\left(A^{*}\right)^{*} \simeq A$ and $\mathbb{F}_{p}[G]^{*} \simeq \mathbb{F}_{p}[G]$ as $\mathbb{F}_{p}[G]$-modules for any finitely 
generated $\mathbb{F}_{p}[G]$-module $A$, from which we see that every finitely generated free $\mathbb{F}_{p}[G]$-module is an injective object in the category of finitely generated $\mathbb{F}_{p}[G]$-modules.

Let $r=\operatorname{dim}_{\mathbb{F}_{p}} E^{G}$. Then we see that $r=\operatorname{dim}_{\mathbb{F}_{p}}\left(E^{G}\right)^{*}=\operatorname{dim}_{\mathbb{F}_{p}}\left(E^{*}\right)_{G}$, and that there exists a surjection of $\mathbb{F}_{p}[G]$-modules

$$
\mathbb{F}_{p}[G]^{\oplus r} \longrightarrow E^{*}
$$

by Nakayama's lemma, from which we obtain an injection.

$$
h: E \longrightarrow \mathbb{F}_{p}[G]^{\oplus r}
$$

by taking the dual $*$. Let $i: E \rightarrow R\left(k^{\mathrm{ur}, p}\right)$ be the natural inclusion map. Since $R\left(k^{\mathrm{ur}, p}\right) \simeq \mathbb{F}_{p}[G]^{\oplus[k: \mathbb{Q}]}$ by $(2), R\left(k^{\mathrm{ur}, p}\right)$ is an injective object in the category of finitely generated $\mathbb{F}_{p}[G]$-modules. Hence there exists a homomorphism $g$ : $\mathbb{F}_{p}[G]^{\oplus r} \longrightarrow R\left(k^{\mathrm{ur}, p}\right)$ such that $g \circ h=i$.

We see that $g$ is injective as follows. Let $y \in(\operatorname{ker} g)^{G}$ be any element. Since $E^{G} \simeq \mathbb{F}_{p}^{\oplus r} \simeq\left(\mathbb{F}_{p}[G]^{\oplus r}\right)^{G}$, the injection $h$ induces the isomorphism $E^{G} \simeq$ $\left(\mathbb{F}_{p}[G]^{\oplus r}\right)^{G}$. Hence $y=h(x)$ for some $x \in E^{G}$ and we have $0=g(y)=i(x)$, which implies $x=0$ and $y=0$. Thus we show that $(\operatorname{ker} g)^{G}=0$, which in turn is equivalent to $\operatorname{ker} g=0$.

Put $P=\operatorname{im}(g)$. Then $P \simeq \mathbb{F}_{p}[G]^{\oplus r}$ and $E \subseteq P$. Let $x \in(P \cap N)^{G}$ be any element. Because, as we have seen, $P^{G}=E^{G}$ and $E \cap N=0$ holds, we have $x=0$. Hence we deduce $P \cap N=0$, then $P+N$ is a direct sum in $R\left(k^{\mathrm{ur}, p}\right)$.

Because any direct factor of a free $\mathbb{F}_{p}[G]$-module is also free, we deduce from (2) that

$$
N \simeq \mathbb{F}_{p}[G]^{\oplus s}
$$

Hence $P+N=P \oplus N$ is also a free $\mathbb{F}_{p}[G]$-module. Then injectivity of $P \oplus N$ shows that the exact sequence of $\mathbb{F}_{p}[G]$-modules

$$
0 \longrightarrow P \oplus N \longrightarrow R\left(k^{\mathrm{ur}, p}\right) \longrightarrow R\left(k^{\mathrm{ur}, p}\right) /(P \oplus N) \longrightarrow 0
$$

splits, which implies that there exists a free $\mathbb{F}_{p}[G]$-submodule $Q$ of $R\left(k^{\mathrm{ur}, p}\right)$ with $R\left(k^{\mathrm{ur}, p}\right)=P \oplus Q \oplus N$.

Now we estimate the $\mathbb{F}_{p}[G]$-rank of $Q$. From the exact sequence

$$
0 \longrightarrow \mathcal{O}_{k^{\mathrm{ur}, p}}^{\times} / \mu_{p} \stackrel{p}{\longrightarrow} \mathcal{O}_{k^{\mathrm{ur}, p}}^{\times} \longrightarrow \mathcal{O}_{k^{\mathrm{ur}, p}}^{\times} / p \longrightarrow 0,
$$

we derive the exact sequence

$$
\mathcal{O}_{k}^{\times} \longrightarrow\left(\mathcal{O}_{k^{\mathrm{ur}, p}}^{\times} / p\right)^{G} \longrightarrow H^{1}\left(G, \mathcal{O}_{k^{\mathrm{ur}, p}}^{\times} / \mu_{p}\right) .
$$

Also, from the exact sequence

$$
0 \longrightarrow \mu_{p} \longrightarrow \mathcal{O}_{k^{\mathrm{ur}, p}}^{\times} \longrightarrow \mathcal{O}_{k^{\mathrm{ur}, p}}^{\times} / \mu_{p} \longrightarrow 0,
$$

we get the exact sequence

$$
H^{1}\left(G, \mathcal{O}_{k^{\mathrm{ur}, p}}^{\times}\right) \longrightarrow H^{1}\left(G, \mathcal{O}_{k^{\mathrm{ur}, p}}^{\times} / \mu_{p}\right) \longrightarrow H^{2}\left(G, \mu_{p}\right)=H^{2}(G, \mathbb{Z} / p) .
$$


Then it follows from (4) and (15) that

$r=\operatorname{dim}_{\mathbb{F}_{p}}\left(\mathcal{O}_{k^{\mathrm{ur}, p}}^{\times} / p\right)^{G} \leq \frac{1}{2}[k: \mathbb{Q}]+\operatorname{dim}_{\mathbb{F}_{p}}\left(H^{1}\left(G, \mathcal{O}_{k^{\mathrm{ur}, p}}^{\times}\right) / p\right)+\operatorname{dim}_{\mathbb{F}_{p}} H^{2}(G, \mathbb{Z} / p)$.

If we let $j: \mathrm{Cl}_{k} \longrightarrow \mathrm{Cl}_{k^{\text {ur }, p}}$ be the natural map from the ideal class group $\mathrm{Cl}_{k}$ of $k$ to that of $k^{\mathrm{ur}, p}$ induced by the inclusion $k \subseteq k^{\mathrm{ur}, p}$, then $H^{1}\left(G, \mathcal{O}_{k^{\mathrm{ur}, p}}^{\times}\right) \simeq$ ker $j$ as well known (see [3] for example). It follows from the principal ideal theorem that ker $j$ is equal to the $p$-primary part of $\mathrm{Cl}_{k}$, which is isomorphic to $\operatorname{Gal}\left(k^{\mathrm{ur}, p} / k\right)^{\mathrm{ab}}=G^{\mathrm{ab}}$ by class field theory. Therefore we find from (6) that

$$
\operatorname{rank}_{\mathbb{F}_{p}[G]} P=r \leq \frac{1}{2}[k: \mathbb{Q}]+d(G)+r(G),
$$

which completes the proof of the lemma since $\operatorname{rank}_{\mathbb{F}_{p}[G]}(P \oplus Q)=[k: \mathbb{Q}]$ by (2).

Lemma 3. Let $M / k^{\mathrm{ur}, p}$ be any finite abelian extension linearly disjoint from the maximal abelian extension of $k^{\mathrm{ur}, p}$ which is unramified outside $p$. Then for any $u \in R\left(k^{\mathrm{ur}, p}\right)$ and $\tau \in \operatorname{Gal}\left(M / k^{\mathrm{ur}, p}\right)$, there exist infinitely many degree one principal prime ideals $\Lambda \mathcal{O}_{k^{\mathrm{ur}, p}}$ of $\mathcal{O}_{k^{\mathrm{ur}, p}}$ such that $\Lambda \mathcal{O}_{k^{\mathrm{ur}, p}}$ is prime to $p,(\Lambda$ $\left.\bmod U\left(k^{\mathrm{ur}, p}\right)^{p}\right)=u$ in $R\left(k^{\mathrm{ur}, p}\right)$ and $\left(\Lambda \mathcal{O}_{k^{\mathrm{ur}, p}, M} / k^{\mathrm{ur}, p}\right)=\tau,\left(*, M / k^{\mathrm{ur}, p}\right)$ being the Artin symbol for $M / k^{\mathrm{ur}, p}$.

Proof. Let $L$ be the maximal elementary abelian $p$-extension of $k^{\mathrm{ur}, p}$ which is unramified outside $p$. We obtain the exact sequence

$$
0 \longrightarrow E \longrightarrow R\left(k^{\mathrm{ur}, p}\right) \stackrel{\rho}{\longrightarrow} \operatorname{Gal}\left(L / k^{\mathrm{ur}, p}\right) \longrightarrow 0
$$

by class field theory and the fact that the class number of $k^{\mathrm{ur}, p}$ is prime to $p$, where $\rho$ is the map induced by the reciprocity map from the idele group of $k^{\mathrm{ur}, p}$ to $\operatorname{Gal}\left(L / k^{\mathrm{ur}, p}\right)$. Let $H$ be the maximal unramified abelian extension of $k^{\mathrm{ur}, p}$ and $\sigma=\rho(u)$. We note that $H, L$ and $M$ are linearly disjoint over $k^{\mathrm{ur}, p}$ because $\left[H: k^{\mathrm{ur}, p}\right]$ is prime to $p$. Then, it follows from the Čebotarev density theorem that there exist infinitely many degree one primes $\mathfrak{L}$ of $k^{\mathrm{ur}, p}$ not lying over $p$ such that $\left(\mathfrak{L}, H / k^{\mathrm{ur}, p}\right)=1,\left(\mathfrak{L}, L / k^{\mathrm{ur}, p}\right)=\sigma^{-1}$ and $\left(\mathfrak{L}, M / k^{\mathrm{ur}, p}\right)=\tau$, which implies that $\mathfrak{L}$ is a principal prime ideal, say, $\mathfrak{L}=\Lambda_{0} \mathcal{O}_{k^{\mathrm{ur}, p}}$, and that $\rho\left(\Lambda_{0} \bmod U\left(k^{\mathrm{ur}, p}\right)^{p}\right)=\sigma$. Therefore, $\left(\Lambda \bmod U\left(k^{\mathrm{ur}, p}\right)^{p}\right)=u$ in $R\left(k^{\mathrm{ur}, p}\right)$ and $\left(\Lambda \mathcal{O}_{k^{\mathrm{ur}, p}, M}, k^{\mathrm{ur}, p}\right)=\left(\mathfrak{L}, M / k^{\mathrm{ur}, p}\right)=\tau$ for a prime element $\Lambda=\Lambda_{0} \varepsilon$ with some $\varepsilon \in \mathcal{O}_{k^{\mathrm{ur}, p}}^{\times}$.

Now we shall show the existence of a prime element $\Lambda \in \mathcal{O}_{k^{\mathrm{ur}, p}}$ with properties (a) and (b) to conclude the proof of Proposition 1.

Let $\left\{\sigma_{1}, \sigma_{2}, \cdots, \sigma_{d}\right\}$ be a system of minimal generators of $G$, where $d=d(G)$ is the generator rank of $G$, and let $P$ and $Q=\bigoplus_{i=1}^{t} \mathbb{F}_{p}[G] q_{i}\left(t=\operatorname{rank}_{\mathbb{F}_{p}[G]} Q\right)$ 
be free $\mathbb{F}_{p}[G]$-submodules of $R\left(k^{\mathrm{ur}, p}\right)$ given by Lemma 2 , where $q_{i}$ 's are free $\mathbb{F}_{p}[G]$-basis of $Q$. Then we find that

$$
t \geq \frac{1}{2}[k: \mathbb{Q}]-d(G)-r(G) \geq d=d(G),
$$

by Lemma 2 and assumption (ii) of the proposition. We let

$$
u=\sum_{i=1}^{d}\left(\sigma_{i}-1\right) q_{i} \in Q \subseteq R\left(k^{\mathrm{ur}, p}\right) .
$$

By virtue of Lemma 3, there exists an integer $\Lambda \in \mathcal{O}_{k^{\mathrm{ur}, p}}$ such that $\Lambda \mathcal{O}_{k^{\mathrm{ur}, p}}$ is a degree one prime not lying over $p$, and that $u=\left(\Lambda \bmod U\left(k^{\mathrm{ur}, p}\right)^{p}\right)$. If we assume that equation (3)

$$
\left(\varepsilon \bmod U^{\prime}\left(k^{\mathrm{ur}, p}\right)\right)+\xi\left(\Lambda \bmod U^{\prime}\left(k^{\mathrm{ur}, p}\right)\right)=0
$$

holds for some $\xi \in \mathbb{F}_{p}[G]$ and $\varepsilon \in \mathcal{O}_{k^{\mathrm{ur}, p},}^{\times}$, then we have $\xi\left(\Lambda \bmod U\left(k^{\mathrm{ur}, p}\right)^{p}\right)+(\varepsilon$ $\left.\bmod U\left(k^{\mathrm{ur}, p}\right)^{p}\right) \in N=\operatorname{ker}\left(R\left(k^{\mathrm{ur}, p}\right) \longrightarrow R^{\prime}\left(k^{\mathrm{ur}, p}\right)\right), \xi\left(\Lambda \bmod U\left(k^{\mathrm{ur}, p}\right)^{p}\right)=\xi u \in$ $Q$, and $\left(\varepsilon \bmod U\left(k^{\mathrm{ur}, p}\right)^{p}\right) \in E \subseteq P$, which implies

$$
\xi\left(\Lambda \bmod U\left(k^{\mathrm{ur}, p}\right)^{p}\right)=\left(\varepsilon \bmod U\left(k^{\mathrm{ur}, p}\right)^{p}\right)=0
$$

by Lemma 2, Hence it follows from $\mathcal{O}_{k^{\mathrm{ur}, p}}^{\times} \cap U\left(k^{\mathrm{ur}, p}\right)^{p}=\left(\mathcal{O}_{k^{\mathrm{ur}, p}}^{\times}\right)^{p}$ that $\varepsilon \in$ $\left(\mathcal{O}_{k^{\mathrm{ur}, p}}^{\times}\right)^{p}$. Also, since

$$
\xi \sum_{i=1}^{d}\left(\sigma_{i}-1\right) q_{i}=\xi u=\xi\left(\Lambda \bmod U\left(k^{\mathrm{ur}, p}\right)^{p}\right)=0,
$$

$\xi\left(\sigma_{i}-1\right)=0$ holds for each $1 \leq i \leq d$, which implies that $\xi(\sigma-1)=0$ for any $\sigma \in G$. Hence we deduce that $\xi \in \mathbb{F}_{p} N_{G}$.

Thus we have shown that the above $\Lambda$ has properties (a) and (b), and that $k^{\prime}=k(\sqrt[p]{\lambda})$ for $\lambda=N_{k^{\mathrm{ur}, p} / k} \Lambda$ is a desired field, which concludes the proof of Proposition 1 .

\section{Preliminary Results from embedding problem of Galois EXTENSION}

In this section, we recall some facts from embedding problem of Galois extension to prove Proposition 2 (consult, for example, [6] for this section).

Let $p$ be any prime number and $K / k$ a finite $p$-extension of number fields of finite degree such that $H:=\operatorname{Gal}(K / k)$ fits the following exact sequence with a finite $p$-group $G$ :

$$
1 \longrightarrow \mathbb{Z} / p \stackrel{\iota}{\longrightarrow} G \stackrel{\pi}{\longrightarrow} H \longrightarrow 1 \text {. }
$$

The Galois extension $L / k$ containing $K$ is called a solution of embedding problem (77) if and only if there exists an isomorphism $\varphi: G \simeq \operatorname{Gal}(L / k)$ such that 
the diagram

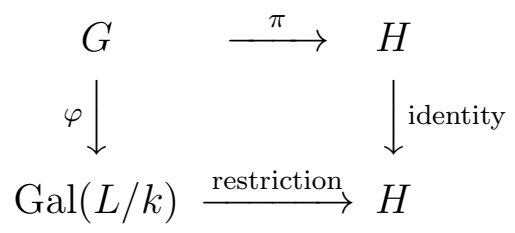

is commutative. In what follows, we assume that $\mu_{p} \subseteq k$. Since the image of $\iota$ is contained the center of $G$ in (7), the $H$-action on the group $\mathbb{Z} / p$ given by (7) is trivial. Hence we shall identify $\mathbb{Z} / p$ with $\mu_{p}$ via an isomorphism $\mathbb{Z} / p \simeq \mu_{p}$ of $H$-modules. Denote by $c \in H^{2}(H, \mathbb{Z} / p)=H^{2}\left(H, \mu_{p}\right)$ the 2-cocycle class associated to group extension (7). Let

$$
0=H^{1}\left(H, K^{\times}\right) \longrightarrow H^{1}\left(H,\left(K^{\times}\right)^{p}\right) \stackrel{\delta}{\longrightarrow} H^{2}\left(H, \mu_{p}\right) \stackrel{l}{\longrightarrow} H^{2}\left(H, K^{\times}\right)
$$

be the exact cohomology sequence derived from the exact sequence of $H$ modules

$$
0 \longrightarrow \mu_{p} \longrightarrow K^{\times} \stackrel{p}{\longrightarrow}\left(K^{\times}\right)^{p} \longrightarrow 0
$$

Then embedding problem (7) has a solution $L / k$ if and only if $c \in \operatorname{im} \delta$, which is equivalent to $l(c)=0$, and a solution $L / k$ is given by as follows: We may assume that $c \neq 0$, because if $c=0$ then (7) is a split extension and $L=K(\sqrt[p]{a})$ is a solution for any $a \in k^{\times}-\left(K^{\times}\right)^{p}$. Let $[f] \in H^{1}\left(H,\left(K^{\times}\right)^{p}\right)$ is the unique 1-cocycle class of the 1-cocycle $f: G \rightarrow\left(K^{\times}\right)^{p}$ such that $\delta([f])=c$. Then 1-cocycle $G \stackrel{f}{\longrightarrow}\left(K^{\times}\right)^{p} \hookrightarrow K^{\times}$is a 1-coboundary by Hilbert's Satz 90 . Hence there exists an element $\alpha \in K^{\times}$such that $\sigma(\alpha) \alpha^{-1}=f(\sigma) \in\left(K^{\times}\right)^{p}$ for any $\sigma \in$ $H$, and $L=K(\sqrt[p]{\alpha})$ gives a solution of (17). Here we note that $L=K(\sqrt[p]{a \alpha})$ is also a solution for any $a \in k^{\times}$since $\sigma(a \alpha)(a \alpha)^{-1}=\sigma(\alpha) \alpha^{-1}=f(\sigma)$ holds.

Conversely, if $L=K(\sqrt{\alpha})$ is a solution of (7), then we see that

$$
\left(\alpha \bmod \left(K^{\times}\right)^{p}\right) \in\left(K^{\times} / p\right)^{H},
$$

since $L / k$ is a Galois extension. If we define the 1-cocycle $f: H \rightarrow\left(K^{\times}\right)^{p}$ by $f(\sigma)=\sigma(\alpha) \alpha^{-1}$, then we have $\delta([f])=c$.

Now we shall give a local condition which assures the existence of a solution of (7). For any prime $\mathfrak{p}$ (including archimedean primes) of $k$, let $H_{\mathfrak{p}}=$ $\operatorname{Gal}\left(K_{\mathfrak{p}} / k_{\mathfrak{p}}\right)$, where $K_{\mathfrak{p}} / k_{\mathfrak{p}}$ is a completion of $K / k$ at a prime of $K$ lying over $\mathfrak{p}$, and we embed $H_{\mathfrak{p}}$ into $H=\operatorname{Gal}(K / k)$ naturally. Put $G_{\mathfrak{p}}=\pi^{-1}\left(H_{\mathfrak{p}}\right) \subseteq G$ and we consider the local embedding problem

$$
1 \longrightarrow \mathbb{Z} / p \longrightarrow G_{\mathfrak{p}} \longrightarrow H_{\mathfrak{p}} \longrightarrow 1 \text {. }
$$

Then $c_{\mathfrak{p}}:=\operatorname{res}_{\mathfrak{p}}(c) \in H^{2}\left(H_{\mathfrak{p}}, \mathbb{Z} / p\right)$ associates the group extension $(7)_{\mathfrak{p}}$, res being the restriction map $H^{2}(H, \mathbb{Z} / p) \longrightarrow H^{2}\left(H_{\mathfrak{p}}, \mathbb{Z} / p\right)$. As in the global case, under the assumption $c_{\mathfrak{p}} \neq 0$, local embedding problem (77) $\mathfrak{p}_{\mathfrak{p}}$ has a solution $L_{\mathfrak{p}} / k_{\mathfrak{p}}$ if and only if $l_{\mathfrak{p}}\left(c_{\mathfrak{p}}\right)=0$, where $l_{\mathfrak{p}}: H^{2}\left(H_{\mathfrak{p}}, \mu_{p}\right) \longrightarrow H^{2}\left(H_{\mathfrak{p}}, K_{\mathfrak{p}}^{\times}\right)$is the map induced by the natural inclusion $\mu_{p} \subseteq K_{\mathfrak{p}}^{\times}$. By class field theory, we have 
the exact commutative diagram

$$
\begin{aligned}
& 0 \longrightarrow H^{2}\left(H, K^{\times}\right) \longrightarrow \bigoplus_{\mathfrak{p}} H^{2}\left(H_{\mathfrak{p}}, K_{\mathfrak{p}}^{\times}\right)
\end{aligned}
$$

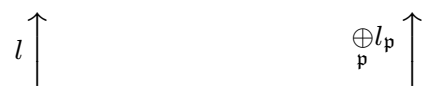

$$
\begin{aligned}
& H^{2}\left(H, \mu_{p}\right) \stackrel{\substack{\oplus \text { res } \\
\longrightarrow}}{\longrightarrow} \bigoplus_{\mathfrak{p}} H^{2}\left(H_{\mathfrak{p}}, \mu_{p}\right)
\end{aligned}
$$

where $\mathfrak{p}$ runs over all the primes of $k$ in the direct sums. Hence we find that $l(c)=0$ if and only if $l_{\mathfrak{p}}\left(c_{\mathfrak{p}}\right)=0$ for all $\mathfrak{p}$, which in turn is equivalent to that local embedding problem $(7)_{\mathfrak{p}}$ has a solution for all the primes $\mathfrak{p}$ of $k$ with $c_{\mathfrak{p}} \neq 0$.

Now we assume that $K / k$ is unramified. Then $H_{\mathfrak{p}}=\operatorname{Gal}\left(K_{\mathfrak{p}} / k_{\mathfrak{p}}\right)$ is a cyclic group for any $\mathfrak{p}$, and $G_{\mathfrak{p}}$ is also cyclic if $c_{\mathfrak{p}} \neq 0$. We note that $H_{\mathfrak{p}}=1$ and $c_{\mathfrak{p}}=0$ if $\mathfrak{p}$ is an archimedean prime. Therefore, if $c_{\mathfrak{p}} \neq 0$, embedding problem $(7)_{\mathfrak{p}}$ has a solution $L_{\mathfrak{p}}$, the unramified extension of $k_{\mathfrak{p}}$ with $\left[L_{\mathfrak{p}}: k_{\mathfrak{p}}\right]=\# G_{\mathfrak{p}}$. Thus we obtain the following:

Theorem $\mathbf{A}$. Let $p$ be a prime number and $K / k$ an unramified $p$-extension of number fields of finite degree. We assume that $\mu_{p} \subseteq k$. Then embedding problem (7) always has a solution.

Remark 1. In fact, we need not assume $\mu_{p} \subseteq k$ in the above theorem.

\section{Proof of Proposition 2}

In this section we shall give a proof of Proposition 2 to complete the proof of Theorems 1 and 2 .

Let $k$ be a number field of finite degree satisfying the assumptions of Proposition 2 for a prime number $p$.

Lemma 4. For any group extension

$$
1 \longrightarrow \mathbb{Z} / p \longrightarrow G^{\prime} \longrightarrow \widetilde{G}_{k}(p) \longrightarrow 1
$$

there exists a finite extension $k^{\prime} / k$ such that

(i) $k^{\text {ur }, p}=k^{\mathrm{ur}, p} k^{\prime}$ and $k^{\prime} \cap k^{\mathrm{ur}, p}=k$ (hence $\widetilde{G}_{k^{\prime}}(p) \simeq \widetilde{G}_{k}(p)$ and every prime of $k^{\prime}$ lying over $p$ splits completely in $k^{\prime \text { ur,p }}$ ),

(ii) there exists an $\varepsilon \in \mathcal{O}_{k^{\prime \text { ur }, p}}^{\times}$such that $k^{\prime \mathrm{ur}, p}(\sqrt[p]{\varepsilon}) / k^{\prime}$ is a Galois extension with $\operatorname{Gal}\left(k^{\prime \text { ur, } p}(\sqrt[p]{\varepsilon}) / k^{\prime}\right) \simeq G^{\prime}$.

Proof. Put $G=\widetilde{G}_{k}(p)$. First we assume that the group extension (8) splits. It follows from the assumption $[k: \mathbb{Q}] \geq 2(2 d(G)+r(G))$ that $\operatorname{dim}_{\mathbb{F}_{p}} \mathcal{O}_{k}^{\times} / p=$ $\frac{1}{2}[k: \mathbb{Q}]>d(G)=\operatorname{dim}_{\mathbb{F}_{p}} G^{\mathrm{ab}} / p$. Hence there exists $\varepsilon \in \mathcal{O}_{k}^{\times}$such that $k(\sqrt[p]{\varepsilon}) / k$ is a cyclic extension of degree $p$ which is linearly disjoint from $k^{\mathrm{ur}, p} / k$. Then we have $\operatorname{Gal}\left(k^{\mathrm{ur}, p}(\sqrt[p]{\varepsilon}) / k\right) \simeq G^{\prime}$. Thus $k^{\prime}=k$ is a desired extension.

Now we assume that group extension (8) does not split. It follows from Theorem A that there exists a Galois extension $L / k$ containing $k^{\mathrm{ur}, p}$ such that $\operatorname{Gal}(L / k) \simeq G^{\prime}$, namely, a solution of embedding problem (8). Let $\alpha \in k^{\mathrm{ur}, p}$ 
be a Kummer generator of $L / k^{\mathrm{ur}, p}: L=k^{\mathrm{ur}, p}(\sqrt[p]{\alpha})$. Since $L / k$ is a Galois extension, we have $\left(\alpha \bmod \left(k^{\mathrm{ur}, p \times}\right)^{p}\right) \in\left(k^{\mathrm{ur}, p \times} / p\right)^{G}$. Hence $\alpha \mathcal{O}_{k^{\mathrm{ur}, p}}=\mathfrak{A}^{p} \mathfrak{a}$ for some ideals $\mathfrak{A}$ of $k^{\mathrm{ur}, p}$ and $\mathfrak{a}$ of $k$. If we denote by $h$ the class number of $k^{\mathrm{ur}, p}$, then $h$ is prime to $p$ and $\alpha^{h} \mathcal{O}_{k^{\mathrm{ur}, p}}=A^{p} \mathfrak{a}^{h}$ for $A \in k^{\mathrm{ur}, p}$ with $A \mathcal{O}_{k_{\mathrm{ur}, p}}=\mathfrak{A}^{h}$. Put $\alpha_{1}=\alpha^{h} A^{-p}$. Then $L=k^{\mathrm{ur}, p}\left(\sqrt[p]{\alpha_{1}}\right)$ and $\alpha_{1} \mathcal{O}_{k^{\mathrm{ur}, p}}=\mathfrak{a}_{1}:=\mathfrak{a}^{h}$ is an ideal of $k$.

Let $p^{e}$ be the exact power of $p$ dividing the order of the ideal $\operatorname{class} \mathrm{cl}_{k}\left(\mathfrak{a}_{1}\right)$ containing $\mathfrak{a}_{1}$ in the class group $\mathrm{Cl}_{k}$ of $k$. By using Proposition 1 repeatedly, we obtain an extension $k^{\prime} / k$ of degree $p^{e}$ such that $k^{\mathrm{ur}, p}=k^{\prime} k^{\mathrm{ur}, p}, k^{\prime} \cap k^{\mathrm{ur}, p}=k$ and $\widetilde{G}_{k^{\prime}}(p) \simeq G$. Denote by $j: \mathrm{Cl}_{k} \longrightarrow \mathrm{Cl}_{k^{\prime}}$ and $N: \mathrm{Cl}_{k^{\prime}} \longrightarrow \mathrm{Cl}_{k}$ be the map induced by the natural inclusion $k \subseteq k^{\prime}$ and the norm map $N_{k^{\prime} / k}$, respectively. Since the natural restriction $\widetilde{G}_{k^{\prime}}(p) \longrightarrow G$, which is an isomorphism, induces the isomorphism $\widetilde{G}_{k^{\prime}}(p)^{\mathrm{ab}} \longrightarrow G^{\mathrm{ab}}$, the order of ker $N$ is prime to $p$ by class field theory. Hence it follows from $N \circ j\left(\operatorname{cl}_{k}\left(\mathfrak{a}_{1}\right)\right)=\operatorname{cl}_{k}\left(\mathfrak{a}_{1}\right)^{p^{e}}$, whose order is prime to $p$, that the order of $j\left(\operatorname{cl}_{k}\left(\mathfrak{a}_{1}\right)\right) \in \mathrm{Cl}_{k^{\prime}}$, say $m$, is also prime to $p$. Then $\mathfrak{a}_{1}^{m}=a^{\prime} \mathcal{O}_{k^{\prime}}$ in $k^{\prime}$ for some $a^{\prime} \in k^{\prime \times}$ and $L k^{\prime}=$ $k^{\prime \mathrm{ur}, p}\left(\sqrt[p]{\alpha_{1}}\right)=k^{\prime \mathrm{ur}, p}\left(\sqrt[p]{\alpha_{1}^{m}}\right)=k^{\prime \text { ur,p }}\left(\sqrt[p]{a^{\prime} \varepsilon}\right)$ for some $\varepsilon \in \mathcal{O}_{k^{\prime \text { ur }, p}}^{\times}$. Therefore we have $\operatorname{Gal}\left(k^{\prime \text { ur, } p}(\sqrt[p]{\varepsilon}) / k^{\prime}\right) \simeq \operatorname{Gal}\left(k^{\prime \text { ur,p }}\left(\sqrt[p]{a^{\prime} \varepsilon}\right) / k^{\prime}\right) \simeq \operatorname{Gal}(L / k) \simeq G^{\prime}$ (see section 4 for the first isomorphism), because $L \cap k^{\prime}=k$ follows from the fact that $k^{\mathrm{ur}, p} \cap k^{\prime}=k$ and the maximal abelian sub-extension of $L / k$ is contained in $k^{\mathrm{ur}, p}$ by the non-splitness of sequence (8) . Thus the above $k^{\prime} / k$ is a desired extension.

Now we shall give a proof of Proposition 2, Let $k^{\prime} / k$ be a finite extension given by Lemma 4 with $\operatorname{Gal}\left(k^{\prime \text { ur,p }}(\sqrt[p]{\varepsilon}) / k^{\prime}\right) \simeq G^{\prime}$ for $\varepsilon \in \mathcal{O}_{k^{\prime u r}, p}^{\times}$and $\widetilde{G}_{k^{\prime}}(p) \simeq$ $\widetilde{G}_{k}(p)$. Put $G=\widetilde{G}_{k^{\prime}}(p)$. We may assume that

$$
\left[k^{\prime}: \mathbb{Q}\right] \geq 2(3 d(G)+r(G))
$$

by replacing $k^{\prime}$ with some suitable finite extension of $k^{\prime}$ given by Proposition 1. Let $R\left(k^{\text {ur }, p}\right)=P \oplus Q \oplus N$ be a decomposition given by Lemma 2. Since $k^{\prime \mathrm{ur}, p}(\sqrt[p]{\varepsilon}) / k^{\prime}$ is a Galois extension and $P$ is a free $\mathbb{F}_{p}[G]$-module $\left(G=\operatorname{Gal}\left(k^{\text {ur }, p} / k^{\prime}\right)\right)$, we have $\left(\varepsilon \bmod U\left(k^{\prime \text { ur }, p}\right)^{p}\right) \in E^{G} \subseteq P^{G}=N_{G} P$. Hence

$$
\left(\varepsilon \bmod U\left(k^{\prime \mathrm{ur}, p}\right)^{p}\right)=N_{G} x
$$

for some $x \in P$.

Let $\left\{\sigma_{1}, \sigma_{2}, \ldots, \sigma_{d}\right\}$ be a generator system of $G$, where $d=d(G)$, and $\left\{q_{1}, q_{2}, \ldots, q_{t}\right\}\left(t=\operatorname{rank}_{\mathbb{F}[G]} Q\right)$ be a system of free basis of the free $\mathbb{F}_{p}[G]$ module $Q: Q=\bigoplus_{i=1}^{t} \mathbb{F}_{p}[G] q_{i}$. Here we note that $t \geq 2 d$ by assumption (9) and $t \geq \frac{1}{2}\left[k^{\prime}: \mathbb{Q}\right]-d(G)-r(G)$

By virtue of Lemma 3, there exists a degree one prime element $\Lambda_{1} \in \mathcal{O}_{k^{\prime} \text { ur }, p}$ prime to $p$ such that

$$
\left(\Lambda_{1} \bmod U\left(k^{\prime \mathrm{ur}, p}\right)^{p}\right)=-x+\sum_{i=1}^{d}\left(\sigma_{i}-1\right) q_{i},
$$


in $R\left(k^{\mathrm{ur}, p}\right)$. If we put $\lambda_{1}=N_{k^{\prime \mathrm{ur}, p} / k^{\prime}} \Lambda_{1}$, then $\lambda_{1}$ is a prime element of $\mathcal{O}_{k^{\prime}}$. It follows from (10) and (11) that every prime of $k^{\prime \text { ur, } p}$ lying over $p$ splits completely and that lying over $\lambda_{1}$ totally ramifies in the degree $p$ extension $k^{\prime \mathrm{ur}, p}\left(\sqrt[p]{\varepsilon \lambda_{1}}\right) / k^{\mathrm{ur}, p}$, respectively. By using Lemma 3 again, we can choose a prime element $\Lambda_{2} \in \mathcal{O}_{k^{\prime} \text { ur }, p}$ prime to $p$ such that

$$
\left(\Lambda_{2} \bmod U\left(k^{\prime \mathrm{ur}, p}\right)^{p}\right)=-x+\sum_{i=1}^{d}\left(\sigma_{i}-1\right) q_{i+d},
$$

and that the prime $\Lambda_{2} \mathcal{O}_{k^{\prime \mathrm{ur}, p}}$ of $k^{\prime \mathrm{ur}, p}$ remains prime in $k^{\prime \mathrm{ur}, p}\left(\sqrt[p]{\varepsilon \lambda_{1}}\right)$. Let $\lambda_{2}=$ $N_{k^{\prime} \mathrm{ur}, p / k^{\prime}} \Lambda_{2}$. Then it follows that $\lambda_{2}$ is also a prime element of $\mathcal{O}_{k^{\prime}}$, and that every prime of $k^{\prime \text { ur, } p}$ lying over $p$ splits completely and that lying over $\lambda_{2}$ totally ramifies in the degree $p$ extension $k^{\prime \text { ur, } p}\left(\sqrt[p]{\varepsilon \lambda_{2}}\right) / k^{\text {ur, } p}$, respectively.

In what follows, we shall show that $k^{\prime \text { ur,p }}\left(\sqrt[p]{\varepsilon \lambda_{1}}, \sqrt[p]{\varepsilon \lambda_{2}}\right)$ is the maximal elementary abelian $p$-extension of $k^{\prime \mathrm{ur}, p}$ which is unramified outside the primes dividing $\lambda_{1} \lambda_{2}$. Assume that $k^{\prime \mathrm{ur}, p}(\sqrt[p]{\beta}) / k^{\prime \mathrm{ur}, p}$ is unramified outside the primes dividing $\lambda_{1} \lambda_{2}$ for $\beta \in k^{\prime \text { ur, } p \times}$. Then we find that

$$
\beta \equiv \eta \prod_{\sigma \in G} \sigma\left(\Lambda_{1}\right)^{a_{\sigma}} \prod_{\sigma \in G} \sigma\left(\Lambda_{2}\right)^{b_{\sigma}} \quad\left(\bmod \left(k^{\prime \mathrm{ur}, p \times}\right)^{p}\right),
$$

for some $\eta \in \mathcal{O}_{k^{\prime \text { ur }, p}}^{\times}$and $a_{\sigma}, b_{\sigma} \in \mathbb{Z}$. Since all the primes of $k^{\prime \text { ur }, p}$ lying over $p$ are unramified in $k^{\prime \mathrm{ur}, p}(\sqrt[p]{\beta}) / k^{\prime \mathrm{ur}, p}$, we have

$$
\begin{aligned}
\left(\eta \bmod U^{\prime}\left(k^{\mathrm{ur}, p}\right)\right) & +\sum_{\sigma \in G} a_{\sigma} \sigma\left(\Lambda_{1} \bmod U^{\prime}\left(k^{\mathrm{ur}, p}\right)\right) \\
& +\sum_{\sigma \in G} b_{\sigma} \sigma\left(\Lambda_{2} \bmod U^{\prime}\left(k^{\text {ur }, p}\right)\right)=0
\end{aligned}
$$

in $R^{\prime}\left(k^{\prime \mathrm{ur}, p}\right)$, which implies

$$
\begin{aligned}
\left(\eta \bmod U\left(k^{\prime \mathrm{ur}, p}\right)^{p}\right) & -\sum_{\sigma \in G}\left(a_{\sigma}+b_{\sigma}\right) \sigma x \\
& +\sum_{\sigma \in G} a_{\sigma} \sigma \sum_{i=1}^{d}\left(\sigma_{i}-1\right) q_{i}+\sum_{\sigma \in G} b_{\sigma} \sigma \sum_{i=1}^{d}\left(\sigma_{i}-1\right) q_{d+i} \in N,
\end{aligned}
$$

in $R\left(k^{\text {ur,p }}\right)$ by (11) and (12). Hence we obtain

$$
\left(\eta \bmod U\left(k^{\prime \mathrm{ur}, p}\right)^{p}\right)-\sum_{\sigma \in G}\left(a_{\sigma}+b_{\sigma}\right) \sigma x=0,
$$

and

$$
\sum_{\sigma \in G} a_{\sigma} \sigma\left(\sigma_{i}-1\right) \equiv \sum_{\sigma \in G} b_{\sigma} \sigma\left(\sigma_{i}-1\right) \equiv 0 \quad(\bmod p) \quad(1 \leq i \leq d)
$$

because $\left(\eta \bmod U\left(k^{\mathrm{ur}, p}\right)^{p}\right)-\sum_{\sigma \in G}\left(a_{\sigma}+b_{\sigma}\right) \sigma x \in P$ and $q_{i}$ 's form a system of free basis of $Q$ over $\mathbb{F}_{p}[G]$. It follows from (14) that there exist some integers $a$ and $b$ such that $a_{\sigma} \equiv a(\bmod p)$ and $b_{\sigma} \equiv b(\bmod p)$ for all $\sigma \in G$, because $\sum_{\sigma \in G} a_{\sigma} \sigma(\tau-1)=\sum_{\sigma \in G} b_{\sigma} \sigma(\tau-1) \equiv 0(\bmod p)$ holds for any $\tau \in G$ by 
(14), which implies $\sum_{\sigma \in G}\left(a_{\sigma} \bmod p\right) \sigma, \quad \sum_{\sigma \in G}\left(b_{\sigma} \bmod p\right) \sigma \in \mathbb{F}_{p} N_{G}$. Hence we derive from (10) and (13) that

$$
\left(\eta \bmod U\left(k^{\mathrm{ur}, p}\right)^{p}\right)-(a+b)\left(\varepsilon \bmod U\left(k^{\mathrm{ur}, p}\right)^{p}\right)=0,
$$

which implies $\eta \equiv \varepsilon^{a+b}\left(\bmod \left(k^{\prime \mathrm{ur}, p \times}\right)^{p}\right)$ by $\mathcal{O}_{k^{\prime \mathrm{ur}, p}}^{\times} \cap U\left(k^{\mathrm{ur}, p}\right)^{p}=\left(\mathcal{O}_{k^{\prime \mathrm{ur}, p}}^{\times}\right)^{p}$. Therefore we conclude that

$$
\beta \equiv \varepsilon^{a+b} \lambda_{1}^{a} \lambda_{2}^{b} \quad\left(\bmod \left(k^{\text {ur }, p \times}\right)^{p}\right),
$$

and that

$$
k^{\prime \mathrm{ur}, p}(\sqrt[p]{\beta})=k^{\prime \mathrm{ur}, p}\left(\sqrt[p]{\left(\varepsilon \lambda_{1}\right)^{a}\left(\varepsilon \lambda_{2}\right)^{b}}\right) \subseteq k^{\prime \mathrm{ur}, p}\left(\sqrt[p]{\varepsilon \lambda_{1}}, \sqrt[p]{\varepsilon \lambda_{2}}\right)
$$

Thus we have shown that $k^{\prime \text { ur, } p}\left(\sqrt[p]{\varepsilon \lambda_{1}}, \sqrt[p]{\varepsilon \lambda_{2}}\right)$ is the maximal elementary abelian $p$-extension of $k^{\prime \mathrm{ur}, p}$ which is unramified outside the primes dividing $\lambda_{1} \lambda_{2}$.

Since the prime $\Lambda_{2} \mathcal{O}_{k^{\prime \mathrm{ur}, p}}$ of $k^{\mathrm{ur}, p}$ remains prime in $k^{\prime \mathrm{ur}, p}\left(\sqrt[p]{\varepsilon \lambda_{1}}\right)$ and ramifies in $k^{\prime \mathrm{ur}, p}\left(\sqrt[p]{\varepsilon \lambda_{2}}\right)$, the decomposition subgroup of $\operatorname{Gal}\left(k^{\prime \mathrm{ur}, p}\left(\sqrt[p]{\varepsilon \lambda_{1}}, \sqrt[p]{\varepsilon \lambda_{2}}\right) / k^{\prime \mathrm{ur}, p}\right)$ at that prime is $\operatorname{Gal}\left(k^{\prime \mathrm{ur}, p}\left(\sqrt[p]{\varepsilon \lambda_{1}}, \sqrt[p]{\varepsilon \lambda_{2}}\right) / k^{\prime \text { ur,p }}\right)$ itself. Hence it follows from Lemma 1 that $k^{\prime \mathrm{ur}, p}\left(\sqrt[p]{\varepsilon \lambda_{1}}, \sqrt[p]{\varepsilon \lambda_{2}}\right)$ has no non-trivial unramified $p$-extensions:

$$
\left(k^{\prime \mathrm{ur}, p}\left(\sqrt[p]{\varepsilon \lambda_{1}}, \sqrt[p]{\varepsilon \lambda_{2}}\right)\right)^{\mathrm{ur}, p}=k^{\prime \mathrm{ur}, p}\left(\sqrt[p]{\varepsilon \lambda_{1}}, \sqrt[p]{\varepsilon \lambda_{2}}\right) .
$$

Also, every prime of $k^{\text {ur, } p}$ lying over $p$ splits completely in $k^{\prime \text { ur,p }}\left(\sqrt[p]{\varepsilon \lambda_{1}}, \sqrt[p]{\varepsilon \lambda_{2}}\right)$.

Now, $K:=k^{\prime}\left(\sqrt[p]{\lambda_{1} \lambda_{2}^{-1}}\right)$ is a desired field as we shall see in the following: We first note that $\operatorname{Gal}\left(k^{\prime \text { ur, } p}\left(\sqrt[p]{\varepsilon \lambda_{1}}\right) / k^{\prime}\right) \simeq \operatorname{Gal}\left(k^{\prime \text { ur,p }}(\sqrt[p]{\varepsilon}) / k^{\prime}\right) \simeq G^{\prime}$ and that every prime of $K$ lying over $p$ splits completely in $k^{\prime \text { ur, } p}\left(\sqrt[p]{\varepsilon \lambda_{1}}, \sqrt[p]{\varepsilon \lambda_{2}}\right) / K$. Since $K / k^{\prime}$ is linearly disjoint from $k^{\prime \text { ur,p }}\left(\sqrt[p]{\varepsilon \lambda_{1}}\right) / k^{\prime}$, we have $\operatorname{Gal}\left(k^{\prime \text { ur,p }}\left(\sqrt[p]{\varepsilon \lambda_{1}}, \sqrt[p]{\varepsilon \lambda_{2}}\right) / K\right) \simeq$ $\operatorname{Gal}\left(k^{\prime \mathrm{ur}, p}\left(\sqrt[p]{\varepsilon \lambda_{1}}\right) / k^{\prime}\right) \simeq G^{\prime}$. Also, because $k^{\prime \mathrm{ur}, p}\left(\sqrt[p]{\varepsilon \lambda_{1}}, \sqrt[p]{\varepsilon \lambda_{2}}\right) / k^{\prime \mathrm{ur}, p}\left(\sqrt[p]{\lambda_{1} \lambda_{2}^{-1}}\right)$ and $k^{\prime \text { ur,p }}\left(\sqrt[p]{\lambda_{1} \lambda_{2}^{-1}}\right) / K$ are unramified, we see that $k^{\prime \text { ur,p }}\left(\sqrt[p]{\varepsilon \lambda_{1}}, \sqrt[p]{\varepsilon \lambda_{2}}\right) / K$ is an unramified $p$-extension. Therefore, it follows from (15) that $k^{\prime \text { ur,p }}\left(\sqrt[p]{\varepsilon \lambda_{1}}, \sqrt[p]{\varepsilon \lambda_{2}}\right)$ is the maximal unramified $p$-extension field over $K$ with Galois group isomorphic to $G^{\prime}$, in which every prime of $K$ lying over $p$ splits completely.

Thus we obtain a desired field $K$. This proves Proposition 2 , hence we have completed the proof of Theorems 1 and 2 .

\section{REFERENCES}

[1] J.-M. Fontaine, B. Mazur, Geometric Galois representations, Elliptic curves, modular forms, $\&$ Fermat's last theorem (Hong Kong, 1993), 41-78, Ser. Number Theory, I, Int. Press, Cambridge, MA, 1995.

[2] A. Fröhlich, Central extensions, Galois groups, and ideal class groups of number fields, Contemporary Mathematics, 24. American Mathematical Society, Providence, RI, 1983.

[3] K. Iwasawa, A note on the group of units of an algebraic number field. J. Math. Pure Appl. 35 (1956), 189-192.

[4] A. Scholz, O. Taussky, Die Hauptideale der kubischen Klassenkörper imaginärquadratischer Zahlkörper: ihre rechnerische Bestimmung und ihr Einfluß auf den Klassenkörperturm, J. Reine Angew. Math. 171 (1934), 19-41.

[5] J. Tate, Global class field theory, Algebraic Number Theory (Proc. Instructional Conf., Brighton, 1965), 162-203, Thompson, Washington, D.C.

[6] K. Uchida, Galois groups of unramified solvable extensions, Tohoku Math. J. 34 (1982), 311-317. 
[7] O. Yahagi, Construction of Number fields with prescribed l-class groups, Tokyo J. of Math. 1 (1978), 275-283.

\author{
Manabu Ozaki, \\ Department of Mathematics, \\ School of Science and Engineering, \\ Kinki University, \\ Kowakae 3-4-1, \\ Higashi-Osaka 577-8502, Japan \\ e-mail: ozaki@math.kindai.ac.jp
}

